\title{
High-Level Performance Evaluation of Object Detection Based on Massively Parallel Focal-Plane Acceleration Requiring Minimum Pixel Area Overhead
}

\author{
Eloy Parra-Barrero ${ }^{1}$, Jorge Fernández-Berni², Fernanda D. V. R. Oliveira ${ }^{3}$, Ricardo Carmona-Galán ${ }^{2}$ \\ and Ángel Rodríguez-Vázquez ${ }^{2}$ \\ ${ }^{1}$ Universidad de Sevilla, 41004 Seville, Spain \\ ${ }^{2}$ Instituto de Microelectrónica de Sevilla (IMSE-CNM), CSIC-Universidad de Sevilla, 41092 Seville, Spain \\ ${ }^{3}$ Universidade Federal do Rio de Janeiro, 21941-901 Rio de Janeiro, Brazil \\ eloyparra_b@hotmail.com,berni@imse-cnm.csic.es,fernanda.dvro@poli.ufrj.br, \{rcarmona,angel\}@imse-cnm.csic.es
}

\begin{abstract}
Keywords: $\quad$ Embedded Systems, Vision Pipeline, Early Vision, Smart Image Sensors, Vision Chips, Focal-Plane Processing, Object Detection, Viola-Jones Algorithm, Performance, Processing Acceleration

Abstract: $\quad$ Smart CMOS image sensors can leverage the inherent data-level parallelism and regular computational flow of early vision by incorporating elementary processors at pixel level. However, it comes at the cost of extra area having a strong impact on the sensor sensitivity, resolution and image quality. In this scenario, the fundamental challenge is to devise new strategies capable of boosting the performance of the targeted vision pipeline while minimally affecting the sensing function itself. Such strategies must also feature enough flexibility to accommodate particular application requirements. From these high-level specifications, we propose a focal-plane processing architecture tailored to speed up object detection via the Viola-Jones algorithm. This architecture is supported by only two extra transistors per pixel and simple peripheral digital circuitry that jointly make up a massively parallel reconfigurable processing lattice. A performance evaluation of the proposed scheme in terms of accuracy and acceleration for face detection is reported.
\end{abstract}

\section{INTRODUCTION}

Unlike CCD, CMOS technologies do permit the co-existence of sensing and processing devices on the same physical substrate. This in turn enables the incorporation of intelligence at different levels within an image sensor chip (Ohta, 2007). In terms of computational efficiency and memory access saving, the most suitable scheme is focal-plane sensing-processing (Zarándy, 2011). As shown in Fig. 1, focal-plane sensing-processing vision chips feature concurrent image capture and low-level processing implemented by an array of interconnected elementary mixed-signal cells, also known as multifunctional pixels. This scheme perfectly adapts to the inherent characteristics of early vision, the most resource-consuming stage of vision pipelines due to huge amount of raw information to be handled. The massively parallel processing and distributed memory provided by focal-plane lattices can thus achieve a drastic reduction of memory accesses and a significant computing acceleration during this critical stage.

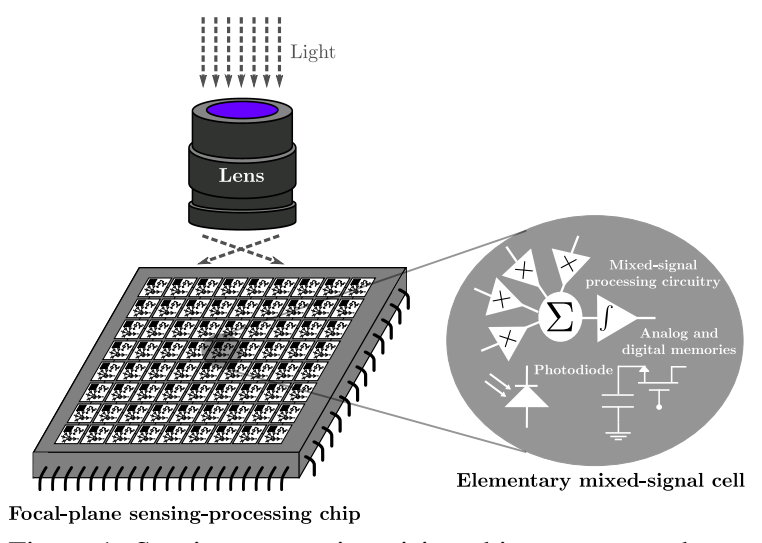

Figure 1: Sensing-processing vision chips capture and preprocess images at the focal plane.

Unfortunately, the incorporation of processing circuitry at pixel level has clashing implications on the other functionality targeted by the chip, image sensing. For a prescribed silicon area-i.e. for a prescribed budget-there is an immediate tradeoff be- 


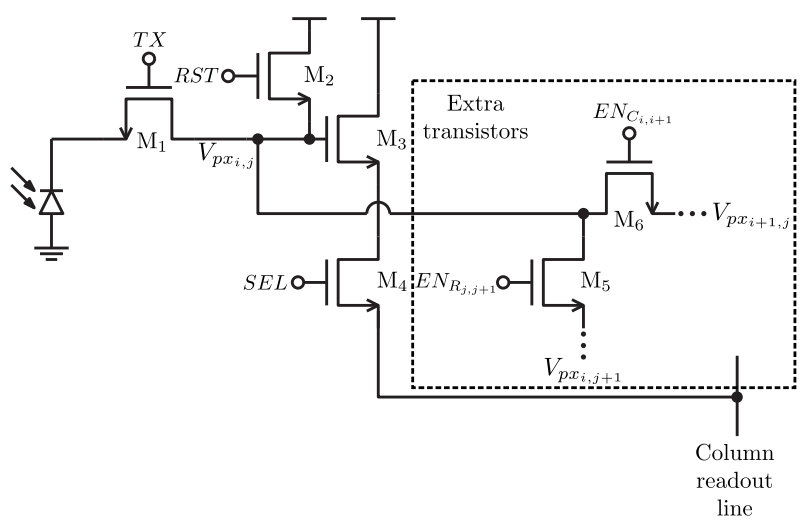

(a)

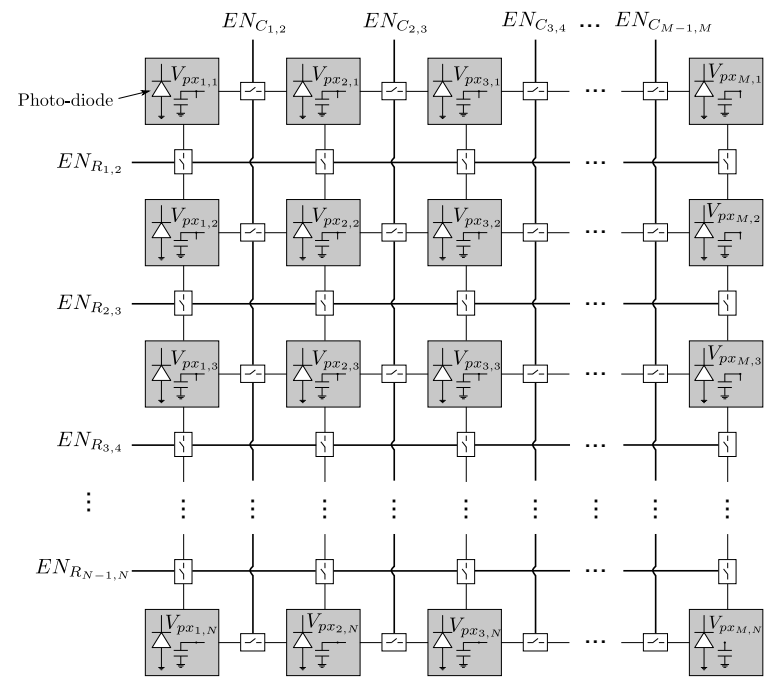

(b)

Figure 2: Proposed pixel circuitry (a), and resulting focal-plane processing lattice (b). The signals $E N_{C_{i, i+1}}$ and $E N_{R_{j, j+1}}$ are driven by the output of corresponding peripheral shift registers.

tween the early vision capabilities embedded into the sensor and the area devoted to capture light per pixel. Likewise, a greater degree of focal-plane smartness accomplished by adding more transistors for processing inevitably leads to a reduction of the image resolution since the pixel size increases. Finally, circuits other than those specifically designed to deal with image capture and readout constitute sources of noise affecting image quality. There is therefore a clear need for circuit structures capable of maximally exploiting the advantages of focal-plane processing while minimally affecting the performance of image sensing.

All in all, this paper presents a focal-plane architecture only requiring two extra transistors per pixel with respect to the standard 3-T/4-T Active Pixel Sensors (APS) that typically make up commercial CMOS image sensors (Fowler, 2015). Additional peripheral digital circuitry enables reconfigurability. We describe how the proposed architecture renders programmable rectangular-wise averaging across multiple image regions in parallel. We then explain why this pre-processing is useful for feature extraction acceleration within the Viola-Jones object detection framework. Lastly, in the context of face detection, we show how a simple re-design of the first stage of the classification cascade in conjunction with the proposed focal-plane pre-processing significantly improves the algorithm throughput with little impact on the detection performance.

\section{FOCAL-PLANE PROCESSING ARCHITECTURE}

A standard 4T APS is depicted in Fig. 2(a) where two minimum-size transistors have been added to play the role of switches. These switches interconnect the node holding the pixel voltage with the same node at neighboring pixels. Their activation is controlled by the corresponding column-wise and rowwise digital signals denoted by $E N_{C_{i, i+1}}$ and $E N_{R_{j, j+1}}$, driven by peripheral shift registers. The resulting focal-plane processing lattice, once the pixel matrix has been composed by aggregation of this elementary cell and removal of unconnected switches at the edges, is shown in Fig. 2(b). Note that the transistors exclusively related to the sensing function in Fig. 2(a), namely $\mathrm{M}_{1-4}$, have been eliminated in this lattice to avoid clutter. The capacitor per pixel represents the capacitance - parasitic or designed ad-hocassociated with the pixel voltage node.

The structure in Fig. 2(b) enables the progressive averaging of rectangular pixel regions in a massively parallel way. This averaging is carried out by setting the adequate interconnection patterns through $E N_{C_{i, i+1}}$ and $E N_{R_{j, j+1}}$. Thus, when the extra switches inserted per pixel are set $\mathrm{ON}$, charge redistribution takes place between the interconnected capacitors. The final voltage after this redistribution is the same for all the pixels making up the considered region, that is, their average value. A key issue concerning this hardwired computation is that no additional energy contribution is required apart from the energy 

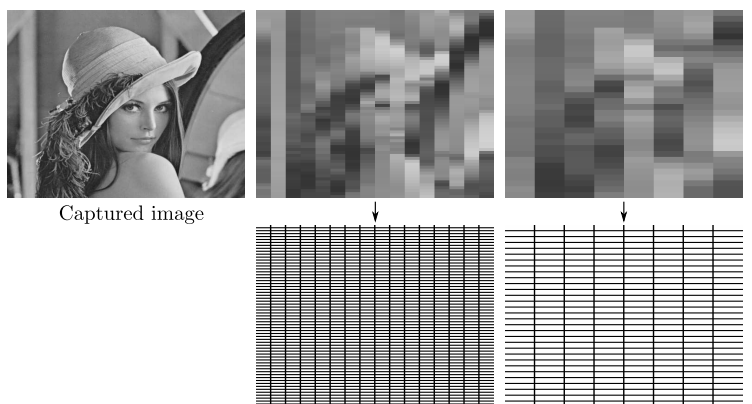

Figure 3: Example of the aggregated pre-processing that can be provided by the proposed focal-plane architecture.

injected into the pixel matrix during the image capture reset (Fernández-Berni et al., 2011). This reset energy is first partly consumed by photo-transducing and then by the dynamics of the charge redistribution, making the whole operation extremely powerefficient. Subsequent interconnection patterns could be established in order to obtain new averaging maps by joining regions averaged just by the previous pattern. This process can continue as requested by the algorithm exploiting it, as long as the unavoidable charge leakage across the chip does not exceed a prescribed limit affecting the precision of the computations. Too many averaging grids could also limit the frame rate since, after each step-image capture, first averaging grid, second averaging grid...-a readout stage must be performed. This is mandatory due to the destructive nature of the processing taking place in every grid with respect to the image representation provided by the previous one. An illustrative example is depicted in Fig. 3, where two consecutive rectangular averaging grids are applied over the original Lena image. Note that only one pixel per rectangle must be readout as all the pixels within a particular region hold the same value. This significantly reduces the number of analog-to-digital conversions with respect to the original captured image. Note also that the rectangles rendered by the second grid come from grouping regions of $2 \times 2$ rectangles from the first grid and then averaging again, thus destroying the previous representation. For every grid, all the signals $E N_{C_{i, i+1}}$ and $E N_{R_{j, j+1}}$ are set to logic '1' but those falling at the boundaries between rectangles that must be set to logic ' 0 ', thereby confining charge redistribution to the desired regions.

\section{VIOLA-JONES ALGORITHM}

The Viola-Jones sliding window object detector (Viola and Jones, 2004) is considered a milestone in real- time generic object recognition. It certainly requires a cumbersome previous training, demanding a large number of cropped samples. But once trained, the detection stage is fast thanks to the computation of the integral image, an intermediate image representation speeding up feature extraction, and to a cascade of classifiers of progressive complexity. Despite its simplicity and detection effectiveness, the algorithm still requires a considerable amount of computational and memory resources in terms of embedded system affordability. Different approaches have been proposed in the literature in order to increase the performance on a limited hardware infrastructure (Camilli and Kleihorst, 2011; Jia et al., 2012; Ouyang et al., 2015). In this paper, we describe a new alternative for the embedded implementation of the algorithm based on processing acceleration from the very beginning of the signal chain, the sensing plane itself.

As just mentioned, feature extraction from the integral image is one of the keys for the success of the Viola-Jones detector. The so-called Haar-like features simply imply contrast comparison of rectangular pixel regions across the sliding window. Some examples are shown in Fig. 4. For each feature, a weighted sum —or average — of the pixels within the white rectangles is subtracted from a weighted sumor average - of the pixels within the black rectangles. The integral image, obtained in one pass over the input image, enables the calculation of these sums by accessing only four of its accumulated pixels instead of massive processing over the original raw pixels. Likewise, contrast normalization for detection in any lighting conditions demands the computation of the squared integral image. This normalization precludes any attempt of skipping the computation of the integral image by directly evaluating the Haarlike features from averaging grids as proposed in Section 2. Furthermore, the large number of features to be extracted-e.g., over 2000 for the OpenCV (Bradski, 2000) baseline implementation of Viola-Jones face detection - would require a great deal of focalplane grids per captured image, impacting the reachable frame rate. Instead, we propose to exploit a reduced number of grids to accelerate the first stage of the classifier. This stage, the most discriminative of the cascade, is designed to rapidly reject windows with very low probability of containing the targeted object. As explained next, it can be re-defined to make use of the averaging grids while requiring neither the integral image nor normalization. Note that a first advantage of this scheme is that the computation of both integral images, needed in any case for the rest of the classifier stages, can be carried out in parallel with the evaluation of the first stage. 


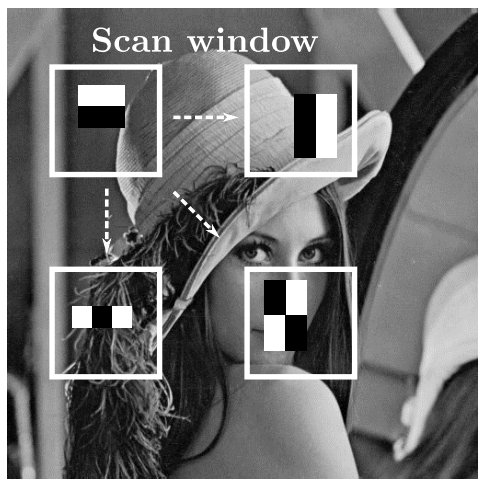

Figure 4: The Haar-like features are evaluated at multiple locations and scales of the input image.
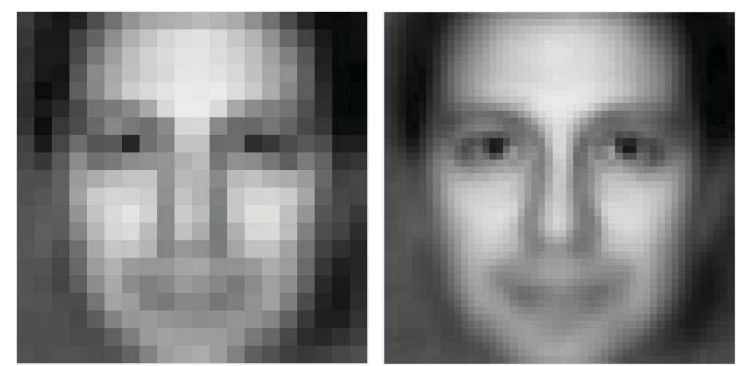

Figure 5: Generic face: $20 \times 20$ pixels (left), $50 \times 50$ pixels (right).

\section{RE-DESIGN OF THE FIRST STAGE}

The re-design described next corresponds to the first stage of the cascade 'haarcascade_frontalface_alt.xml' provided by OpenCV, release 2.4.10 for Windows operating systems. This cascade includes 21 stages and is defined for a minimum detection size of $20 \times 20$ pixels. The ViolaJones face detector provided by the same release is our baseline implementation of the algorithm. As a test bench, we use the Caltech Frontal Face Dataset (Weber, 1999). It includes 450 frontal face images of 27 people under different lighting, expressions and backgrounds with a resolution of $896 \times 592$ pixels in JPEG format. In order to work with a representative face allowing subsequent extrapolation to the rest of the dataset, a 'generic face' was first created. To this end, we ran the algorithm over the entire dataset. All detected faces were scaled to a common size and then averaged. The resulting face is shown in Fig. 5 for two different resolutions.

The three features composing the first stage of the cascade are depicted in Fig. 6. The evaluation of each of these features entails the comparison with a specific threshold coming from the training process that

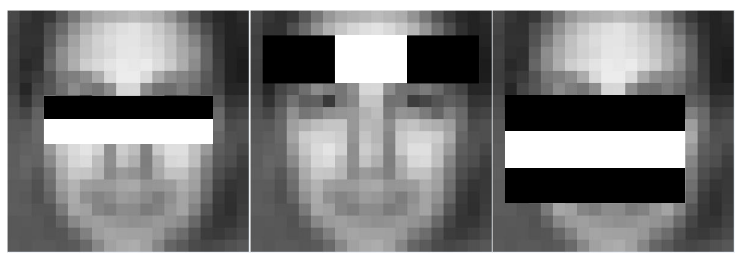

Figure 6: Features composing the first stage of the cascade 'haarcascade_frontalface_alt.xml' provided by OpenCV.

Table 1: Checking only the sign of the features instead of numerical comparison with thresholds leads to a much more discriminative first stage of the Viola-Jones face detection algorithm.

\begin{tabular}{ccc}
\hline & Thresholds & Only sign \\
\hline True-positives & 445 & 437 \\
\hline False-positives & 7 & 6 \\
\hline False-negatives & 5 & 13 \\
\hline Precision & $98.5 \%$ & $98.7 \%$ \\
\hline Recall & $98.9 \%$ & $97.1 \%$ \\
\hline $\begin{array}{c}\text { Rejected windows } \\
\text { at first stage }\end{array}$ & $28.9 \%$ & $66.7 \%$ \\
\hline Execution time & $86.3 \mathrm{~s}$ & $41.9 \mathrm{~s}$ \\
\hline
\end{tabular}

generated the cascade. There is also a threshold associated with the stage itself. For an adequate numerical comparison, these thresholds demand the normalization of the input image by using the squared integral image, as previously mentioned. A remarkable first result stems from removing this threshold analysis for the first stage. Instead, we simply check the sign (Abramson et al., 2007) of the three features, only accepting for further processing those windows where the three of them produce a positive outcome. Otherwise, the window is rejected. As a result, a much more discriminative first stage is achieved. Table 1 summarizes the performance of the algorithm when threshold comparison is carried out and when only the sign is checked. The detector is configured to detect faces with a minimum size of $80 \times 80$ pixels. The scale factor for the sliding window is set to the default value, 1.1. Notably, the values of precision and recall (Klette, 2014) remain very similar in both cases but the percentage of rejected windows when only the sign is checked is significantly larger. The time required by the algorithm to process the entire test bench-on an Intel Core i7 at $2.4 \mathrm{GHz}$ - is thus reduced by more than $50 \%$.

Sign checking instead of threshold comparison therefore permits to skip the computation of the squared integral image for the first stage of the cas- 


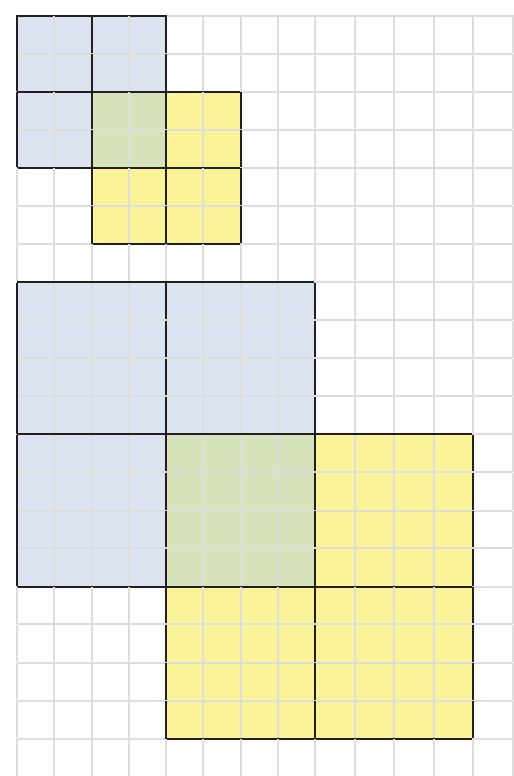

Figure 7: Each focal-plane grid is rendered by grouping rectangles from the previous one.

cade. We can also get rid of the integral image for this stage by having the rectangular regions making up each feature computed from the massively parallel focal-plane processing described in Section 2. There is however a major limitation for this approach due to the aggregated nature of the operation. Keep in mind that each grid is always formed by grouping rectangles of the previous one. This forces a minimum step of scaling and shifting for the sliding window according to the elementary rectangle of the first grid. An example is depicted in Fig. 7. It can be seen that a first scale featuring a $2 \times 2$-px elementary rectangle necessarily leads to a second scale with a $4 \times 4$-px elementary rectangle-we are assuming that both image dimensions are scaled in the same way. Likewise, the shifting step of the sliding window is 2-pixel for the first scale and consequently 4-pixel for the second scale.

In order to overcome this limitation, we propose the definition of new features for the first stage that take into account the coarse pixel aggregation at the focal plane. To this end, we have defined an elementary grid made up of $4 \times 4$-px rectangles over the 'generic face', as shown in Fig. 8(a). An estimate of the most stable regions to define new features over this grid can be obtained by computing the difference between the 'generic face' and each of its constituent faces detected from the test bench, followed by the average of the absolute values of these differences. The normalized result is depicted in Fig. 8(b). Black indicates no variability at all whereas white repre-

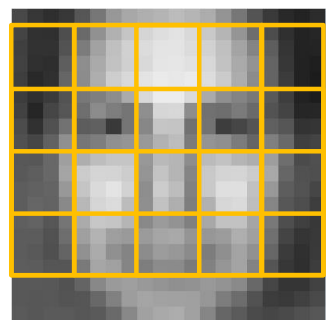

(a)

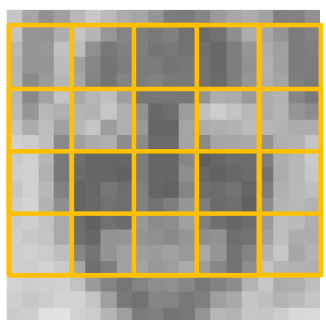

(b)
Figure 8: (a) Basic grid defined over the 'generic face'; (b) Variability of the 'generic face' with respect to its constituent faces detected from the test bench.

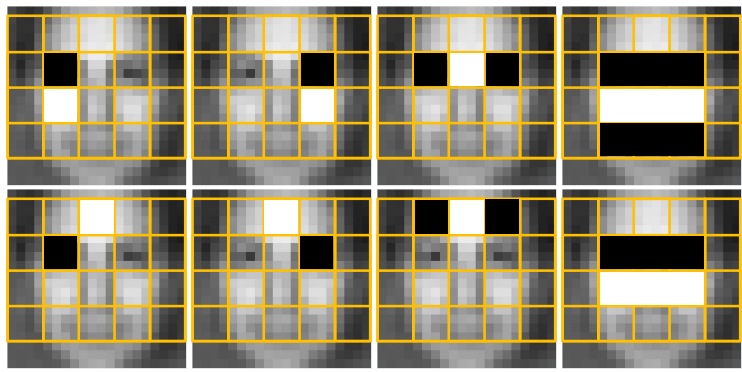

Figure 9: Features defining the new first stage of the algorithm over the basic grid.

sents maximum variability. We can conclude from this figure that the nose, forehead and cheek areas are good candidates for such regions. Conversely, eyes and mouth present significant variability among faces. For the eyes, this variability is compensated by their usual high contrast with other zones of the face. All in all, we have carried out a heuristic search of discriminative features defined over the basic grid in Fig. 8(a). The resulting features, represented in Fig. 9, are those which best ranked in terms of highest number of successful sign checks over the faces detected when running the baseline algorithm. The performance of this baseline implementation when the features of the original first stage of the cascade are substituted for those of Fig. 9 is reported in Table 2. The detector is configured in the same way as in Table 1 and ran on the same PC. The new features achieve much greater discrimination while still impacting little on the detection performance. The execution time is decreased by an additional $50 \%$ with respect to only checking the sign of the original features. 
Table 2: The new features represented in Fig. 9 achieve even further discrimination at the first stage with little impact on the detection performance.

\begin{tabular}{cc}
\hline & $\begin{array}{c}\text { Only sign } \\
\text { (new features) }\end{array}$ \\
\hline True-positives & 427 \\
\hline False-positives & 10 \\
\hline False-negatives & 23 \\
\hline Precision & $97.7 \%$ \\
\hline Recall & $94.9 \%$ \\
\hline $\begin{array}{c}\text { Rejected windows } \\
\text { at first stage }\end{array}$ & $93.7 \%$ \\
\hline Execution time & $21.0 \mathrm{~s}$ \\
\hline
\end{tabular}

\section{PERFORMANCE EVALUATION UNDER CHIP OPERATION CONDITIONS}

The figures in Table 2, while interesting on their own, do not reflect the targeted real operation conditions yet. The features were certainly defined according to the restrictions imposed by the elementary grid, but the scale factor was still set to 1.1 for comparison with Table 1. As mentioned in Section 4, such scale factor cannot be provided by the focal-plane acceleration sketched in Fig. 2. The detector must therefore be configured in order to faithfully emulate the scaling and shifting of the sliding window according to the scheme in Fig. 7. This implies to scale the input frame following the geometric progression 4 (elementary grid), $8,16,32 \ldots$ This scale progression is depicted in Fig. 10(a) for the Lena image. The sliding window must also be consequently shifted at each scale. We must emphasize again at this point that only one pixel per rectangle needs to be readout. This is crucial to reduce the speed-and thereby the power-requirements of an analog-to-digital conversion stage capable of coping with each scale of the sequence in proper timing. The performance of the algorithm under these conditions is extremely poor, as shown along the column 'Single scaling progression' in Table 3. The pixel aggregation is too coarse for the algorithm to carry out a reliable detection. In order to circumvent this problem, we must introduce a second scaling — and shifting — progression, namely $6,12,24,48 \ldots$ This second progression is not compatible with the first one for a per-frame processing owing to the destructive nature of the focal-plane processing described in Section 2. Both progressions must therefore be alternated throughout the input im-

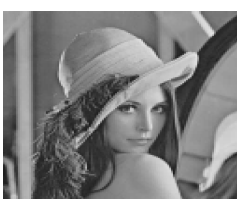

$4 \times 4$-px grid

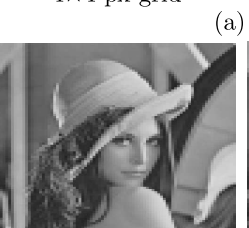

$6 \times 6$-px grid

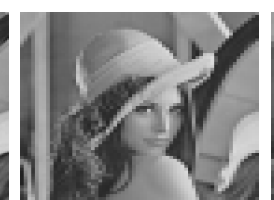

$8 \times 8$-px grid First scaling progression

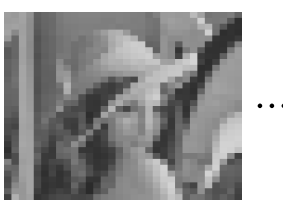

$16 \times 16$-px grid

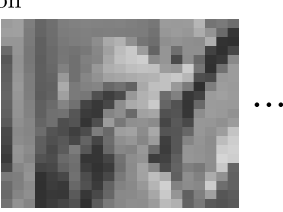

$24 \times 24-p x$ grid (b) Second scaling progression

Figure 10: Scaling progressions that must be alternatively applied to the frames of a sequence according to the image representations attainable from the proposed focal-plane processing architecture.

Table 3: A single progression of focal-plane grids leads to poor performance. Instead, two alternate scaling progressions achieve an excellent performance in terms of execution time.

\begin{tabular}{ccc}
\hline & $\begin{array}{c}\text { Single scaling } \\
\text { progression }\end{array}$ & $\begin{array}{c}\text { Alternate scaling } \\
\text { progressions }\end{array}$ \\
\hline True-positives & 207 & 426 \\
\hline False-positives & 0 & 12 \\
\hline False-negatives & 243 & 24 \\
\hline Precision & $100 \%$ & $97.2 \%$ \\
\hline Recall & $46.0 \%$ & $94.7 \%$ \\
\hline Execution time & $3.4 \mathrm{~s}$ & $5.7 \mathrm{~s}$ \\
\hline
\end{tabular}

age sequence. Note that this condition assumes slow, or moderate at most, motion of the targeted object-a face in this case-across the scene for successful detection. Otherwise, the successive focal-plane grids applied over one frame would be uncorrelated with those applied on the next frame. Typical applications like face recognition-demanding previous face detection - or automatic camera focus based on face detection should meet this constraint in most cases. The performance of the algorithm when the two proposed scaling progressions are applied over each image of the test bench is summarized along the column 'Alternate scaling progressions' in Table 3 . The values of precision and recall are slightly worse than those of the original algorithm in Table 1 but the execution time has been reduced by one order of magnitude. This is achieved thanks to a much more discriminative first stage in conjunction with a coarser but effective strategy of scaling and shifting of the sliding window. 


\section{CONCLUSIONS}

We have described a massively parallel focal-plane processing architecture capable of rendering useful image representations for object detection acceleration. This is accomplished while minimally impacting the pixel size and the detection efficiency. These features, together with reconfigurability, are instrumental for the potential commercial exploitation of focalplane sensing-processing chips. Future work will be focused on the physical design of the chip itself. In addition to the circuit blocks described in this paper, the analog-to-digital conversion stage will also have to be carefully addressed for a timely readout of the captured image and successive processing grids.

\section{ACKNOWLEDGEMENTS}

This work has been funded by the Spanish Government through projects TEC2012-38921-C02 MINECO (European Region Development Fund, ERDF/FEDER), Junta de Andalucía through project TIC 2338-2013 CEICE and by the Office of Naval Research (USA) through grant N000141410355.

\section{REFERENCES}

Abramson, Y., Steux, B., and Ghorayeb, H. (2007). Yet even faster (YEF) real-time object detection. Int. J. of Intelligent Systems Technologies and Applications, 2(2-3):102-112.

Bradski, G. (2000). The OpenCV library. Dr. Dobbs Journal of Software Tools.

Camilli, M. and Kleihorst, R. (2011). Demo: Mouse sensor networks, the smart camera. In 5th ACM/IEEE Int. $C$. on Distributed Smart Cameras, Ghent, Belgium.

Fernández-Berni, J., Carmona-Galán, R., and CarranzaGonzález, L. (2011). FLIP-Q: A QCIF resolution focal-plane array for low-power image processing. IEEE Int. Journal of Solid-State Circuits, 46(3):669680.

Fowler, B. (2015). Solid-state image sensors. In Kriss, M., editor, Handbook of Digital Imaging. John Wiley \& Sons, Ltd.

Jia, H., Zhang, Y., Wang, W., and Xu, J. (2012). Accelerating viola-jones face detection algorithm on GPUs. In IEEE Int. Conf. on Embedded Software and Systems, pages 396-403.

Klette, R. (2014). Concise Computer Vision. Springer.

Ohta, J. (2007). Smart CMOS Image Sensors and Applications. CRC Press.

Ouyang, P., Yin, P., Yin, S., Zhang, Y., Liu, L., and Wei, S. (2015). A fast integral image computing hardware architecture with high power and area efficiency. IEEE Transactions on Circuits and Systems II, 62(1):75-79.

Viola, P. and Jones, M. (2004). Robust real-time face detection. Int. J. of Computer Vision, 57(2):137-154.

Weber, M. (1999). Caltech frontal face dataset. http://www.vision.caltech.edu/htmlfiles/archive.html.

Zarándy, A., editor (2011). Focal-plane Sensor-Processor Chips. Springer. 